\title{
OGT controls mammalian cell viability by regulating the proteasome/mTOR/ mitochondrial axis
}

Xiang Li ${ }^{1,2 \#}$, Xiaojing Yue ${ }^{1 \#}$, Hugo Sepulveda ${ }^{1}$, Rajan A. Burt ${ }^{3}$, David A. Scott ${ }^{4}$, Steven A. Carr ${ }^{3}$, Samuel A. Myers $^{1 *}$, Anjana Rao ${ }^{1,2,5 *}$

${ }^{1}$ Division of Signaling and Gene Expression, La Jolla Institute for Immunology, La Jolla, CA, USA.

${ }^{2}$ Sanford Consortium for Regenerative Medicine, La Jolla, CA, USA.

${ }^{3}$ The Broad Institute of MIT and Harvard, Cambridge, MA, USA.

${ }^{4}$ Cancer Metabolism Core, Sanford Burnham Prebys Medical Discovery Institute, La Jolla, CA, USA.

${ }^{5}$ Department of Pharmacology and Moores Cancer Center, University of California, San Diego, La Jolla, CA, USA.

\#These authors contributed equally to this work.

* Correspondence: sam@lji.org (S.A.M.); arao@lji.org (A.R.) 


\section{Abstract}

O-GlcNAc transferase (OGT) is an essential X-chromosome-encoded enzyme that catalyzes the addition of $\mathrm{N}$ acetylglucosamine (GlcNAc) to the hydroxyl groups of serine and threonine residues on many nuclear and cytosolic proteins. This posttranslational modification is reversible and is actively removed by the $O$ GIcNAc'ase OGA. It was shown more than two decades ago that OGT is essential for mammalian cell viability, but the underlying mechanisms are still enigmatic. Given the close association between OGT and human diseases, such as cancer, diabetes and cardiovascular disease, identification of the mechanisms by which OGT controls cell viability will facilitate the development of therapeutic strategies to manipulate OGT activity. Here, we employ a genome-wide CRISPR-Cas9 viability screen in mouse embryonic stem cells (mESCs) with inducible Ogt deletion to show that the block in cell viability induced by Ogt-deficiency stems from a deleterious increase in mitochondrial oxidative phosphorylation (OXPHOS). Mechanistically, we demonstrate that OGT safeguards mTOR (mechanistic target of rapamycin) activity to maintain mitochondrial fitness through modulation of proteasome activity and intracellular amino acid homeostasis. In the absence of OGT, increased proteasome activity results in increased steady-state amino acid levels, which in turn promote mTOR translocation and activation and increased oxidative phosphorylation. This mechanism also operates in CD8 ${ }^{+} \mathrm{T}$ cells, indicating its generality across mammalian cell types. Genome-wide proteomic and phosphoproteomic analyses show extensive changes in global signaling and confirm our finding of mTOR hyperactivation in OGTdeficient cells. In sum, our study highlights a novel function for OGT in regulating the proteasome/ mTOR/ mitochondrial axis in a manner that maintains homeostasis of intracellular amino acid levels, mitochondrial fitness and cell viability. Since many of the proteins involved in proteasome, mTOR and mitochondrial activity are aberrantly expressed in cancer, and since inhibitors for proteasome and mTOR have been used in cancer therapy, manipulating OGT activity may have therapeutic potential in diseases in which this signaling axis is impaired.

Keywords: Genome-wide CRISPR/Cas9 screen; mouse embryonic stem cells; mechanistic target of rapamycin; ubiquitin-dependent protein degradation; amino acids 


\section{Introduction}

OGT (O-GlcNAc transferase) is an essential X-chromosome-encoded enzyme that catalyzes the addition of $\mathrm{N}$-acetylglucosamine (GlcNAc) to the hydroxyl groups of serine and threonine residues on many nuclear and cytosolic proteins ${ }^{1,2}$. This posttranslational modification is reversible and is actively removed by the $O$-GlcNAc'ase $O_{G A}^{1,2}$. Deletion of the single Ogt gene in the mouse germline results in embryonic lethality prior to blastocyst implantation ${ }^{3,4}$. Although invertebrates such as $C$. elegans and Drosophila can survive without OGT, OGT is essential for the proliferation and survival of essentially all mammalian cell types tested, including mouse embryonic stem cells (mESCs) $)^{1,3}$.

The mechanistic target of rapamycin (mTOR) is a serine/ threonine kinase that regulates fundamental cellular processes including cell growth and metabolism in response to environmental and intracellular signals $^{5,6}$. mTOR is the catalytic subunit of two functionally distinct protein complexes, mTOR complex 1 (mTORC1, rapamycin-sensitive) and mTORC2. It has been reported that $\mathrm{mTORC} 1$ regulates mitochondrial functions by promoting the translation of a subset of nucleus-encoded mitochondrial genes through inhibition of 4E-BPs (eukaryotic translation initiation factor 4E-binding proteins) ${ }^{7}$ or through transcriptional control of mitochondrial regulators such as PGC-1a (PPARy coactivator $1 \alpha)^{8}$.

mTORC1 has been described as a "coincidence detector" which is activated at the lysosomal surface only when both growth factors and nutrients are present ${ }^{5}$. mTORC1 activation requires two separate steps that occur at the lysosomal membrane ${ }^{5,6}$. Growth factor and cellular stress signals induce activation of the small GTPase Rheb, which is located on the lysosomal membrane and directly stimulates mTOR kinase activity in its GTP-bound form. However, mTORC1 only localizes in the vicinity of GTP-bound Rheb when nutrient signals such as amino acids are available to activate the Rag complex, a heterodimer of the small GTPases, RagA and RagC (or RagB and RagD). When RagA (or RagB) are GTP-bound and RagC (or RagD) are bound to GDP, the Rag heterodimers recruit mTORC1 to the lysosomal membrane. The Rag heterodimer is itself activated and anchored to the lysosomal membrane by the pentameric Ragulator complex, a non-canonical guanine nucleotide exchange factor that contains Lamtor 1-5,10. mTOR indirectly senses amino acid levels through proteins associated with the Rag and Ragulator complexes. Overall, high amino acid levels result in increased mTOR activity through a complex network of activating and inhibitory protein-protein interactions that are not yet fully understood ${ }^{5,6,9}$.

To investigate why OGT is essential for the survival of proliferating cells ${ }^{1}$, we generated mouse embryonic stem cells (mESC) with inducible deletion of the Ogt gene. A genome-wide CRISPR-Cas9 viability screen for small guide RNAs (sgRNAs) that rescued the proliferation of Ogt-deleted mESC revealed that the enriched sgRNAs targeted a large number of genes related to mitochondrial function, as well as genes whose products were involved in amino acid sensing by mTOR. We show that Ogt deficiency blocks mammalian cell viability at least partly by increasing proteasome activity and intracellular amino acid levels, which in turn promote the lysosomal translocation and activation of mTORC1. The net result is a deleterious increase in mitochondrial oxidative phosphorylation. By highlighting the biochemical connection between OGT deficiency and high aberrant activation of proteasome function and the mTOR pathway, our studies provide mechanistic insights into the role of $O$-GlcNAc modifications in intracellular homeostasis. 


\section{Results}

\section{Inducible deletion of Ogt impairs cell viability in mESCs}

To overcome the lethality of Ogt deletion in mouse embryonic stem cells (mESC) ${ }^{3}$, we generated conditional Ogt floxed mice homozygous for knock-in alleles of Cre-ERT2 or LSL-YFP in the Rosa26 locus. We crossed

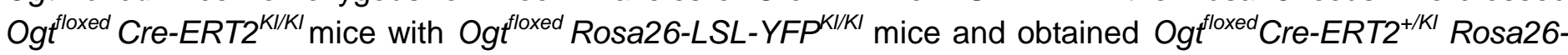
$L S L-Y F P^{+/ K l}$ (hereafter termed Ogt fl) blastocysts (embryonic day 3.5) to derive Ogt $\mathrm{fl}$ mESC lines (Supplementary Fig. 1a). Subsequent experiments were performed with male Ogt $f l \mathrm{mESCs}$ to maximize the likelihood of complete Ogt gene deletion in response to 4-OHT treatment.

At day 6 after 4-OHT addition, the extent of Ogt gene deletion was >95\% as assessed by qRT-PCR for Ogt mRNA (Supplementary Fig. 1b) and the deletion at protein level was nearly complete as assessed by western blot (Supplementary Fig. 1c). Immunostaining confirmed that both OGT and its catalytic product, the O-GlcNAc modification, were almost completely eliminated in the Ogt-deleted cells (hereafter termed Ogt iKO mESC; $i$ for "inducible") (Supplementary Fig. 1d). Ogt iKO mESCs underwent a significant morphology change, from large, tightly packed colonies in the case of $\mathrm{Ogt} f l \mathrm{mESC}$ to small, loosely distributed colonies after Ogt deletion (Fig. 1a); they also displayed a significant impairment of growth rate (Fig. 1b). The impaired growth rate was due specifically to Ogt deletion, since it was rescued by overexpression of wildtype (WT) OGT in Ogt iKO mESCs, concomitantly with restoration of O-GlcNAc staining (Supplementary Fig. 2a, b).

\section{A genome-wide CRISPR-Cas9 screen identifies key regulators of cell survival in OGT-deficient mESCs}

To identify genes whose deletion could restore cell proliferation and increase the survival of Ogt iKO mESC, we conducted an unbiased genome-wide CRISPR-Cas9 viability screen. We stably expressed Cas9 in Ogt $f l$ mESCs, and lentivirally transduced them with the sgRNA Brie library ${ }^{11}$ which contains a pool of 78,637 sgRNAs targeting 19,647 genes with 4 sgRNAs per gene and 1000 control non-targeting sgRNAs. After puromycin treatment for 7 days to select for cells stably expressing the sgRNAs, the cells were split into two groups, an untreated (Ogt fl) group that served as the control, and a second group treated with 4-OHT for 6 days to induce Ogt deletion (Ogt $i K O$ ). On day 13, YFP-negative cells from the control Ogt $f l$ group and $\mathrm{YFP}^{+}$ cells from the Ogt iKO group were sorted and the enriched sgRNAs were identified by next generation deep sequencing (Fig. 1c). The sequencing data were analyzed using the PinAPL-Py platform ${ }^{12}$.

We performed two independent screens that were sequenced to a depth of $>10 \mathrm{M}$ mapped reads per sample, with $>98 \%$ of the genes targeted by the sgRNAs represented in each case (Supplementary Fig. 3a). The volcano plot showed significantly enriched sgRNAs in green and control non-targeting sgRNAs which are not significantly enriched in orange (Supplementary Fig. 3b); the overlap of enriched sgRNAs and their rank in each replicate was shown in Supplementary Fig. 3c. Gene scores were calculated considering the log fold changes of all sgRNAs targeting each gene and the number of sgRNAs targeting each gene that reached statistical significance ${ }^{12}$. In the two replicate experiments, 115 hits were identified by the overlap of sgRNAs enriched in the Ogt iKO groups compared to the control Ogt $f l$ groups ( $p$-value < 0.01) (Supplementary Fig. 3d). Hits with the highest scores, including Hmbs, Mrpl50, Gtpbp8, Socs3 and Opa1 are highlighted (Fig. 1d).

Gene ontology analysis of the 115 candidate hits showed that over 80 hits were annotated as related to the mitochondrion or the mitochondrial respiratory chain complex (Fig. 1e). To further identify the correlations among the candidate hits, we integrated the 115 screen hits against protein-protein interaction (PPI) databases to create a physical network using Metascape, which applies an algorithm called MCODE (Molecular Complex Detection) to identify protein complexes $^{13}$ (Fig. 1f). We identified three MCODE complexes: respiratory electron transport (shown in red), including NADH:ubiquinone oxidoreductase family (Nduf) proteins in mitochondrial complex I; proteins such as mitochondrial ribosomal proteins (Mrpl) involved in mitochondrial translation (shown in blue), and mTOR signaling (shown in green), specifically Rraga and Lamtor2-4 (Fig. 19). 
Metascape analysis also identified protein complex involved in heme biosynthesis (Hmbs, Uros and Urod), quinone biosynthesis (Coq4, Coq5, Coq6 and Coq8b) and iron-sulfur cluster assembly (Glrx5, Isca2 and lba57) (Fig. 1f). Screen hits related to mitochondria and mTOR signaling are considered in detail below. Other candidate hits are being investigated further as part of a separate project.

\section{OGT deficiency results in mitochondrial dysfunction}

To follow up on the large number of enriched sgRNAs that targeted mitochondrial proteins, we assessed mitochondrial function in control and Ogt-deleted cells by evaluating mitochondrial oxidative phosphorylation (OXPHOS). Basal and maximal oxygen consumption rates (OCR), an indicator of OXPHOS, were aberrantly increased in Ogt iKO compared to Ogt fl mESCs, (Fig. 2a). Consistent with the increase in OXPHOS, the mitochondrial complex I inhibitor Rotenone partially rescued the decreased proliferation of Ogt iKO mESCs, despite its substantial toxicity for Ogt $\mathrm{fl}$ mESCs (50\% decrease in cell numbers) (Fig. 2b).

We used an all-in-one CRISPR/Cas9 vector system to individually interrogate the effects of enriched sgRNAs that targeted eight of the top candidate genes identified in our screen (Fig. 1d). Among them were genes encoding mitochondrial proteins (Opa1, Gtpbp8, Mrp/50), proteins involved in heme biosynthesis (Hmbs, Uros) and proteins involved in other cellular functions (Socs3, Xpo5, Ythdc1). Each gene was efficiently deleted as assessed by qRT-PCR (Supplementary Fig. 4a). Transduction with Cas 9 and sgRNAs targeting these genes improved the morphology and growth rate of Ogt iKO mESCs, leading to the formation of large, tightly packed colonies (Fig. 2c and Supplementary Fig. 4b) as well as an $\sim 3$ - to 7-fold increase in cell numbers; however, sgRNAs against $\mathrm{Hmbs}$ and Uros diminished the proliferation of Ogt $\mathrm{fl} \mathrm{mESC}$ and were not further considered (Fig. 2d). In contrast, non-targeting control sgRNAs and sgRNAs targeting Tet1 or Tet2 (genes expressed in ES cells, but whose sgRNAs were not enriched in our screen) did not prevent the dramatic decrease in cell numbers observed upon Ogt deletion (Fig. 2d).

We asked whether sgRNAs targeting the top three candidates, Socs3, Xpo5 and Gtpbp8, affected mitochondrial function. Indeed, sgRNAs targeting each of these three candidate genes reduced the aberrant increase of oxygen consumption rates in Ogt iKO mESCs to normal levels (Fig. 2e-g). Socs3 is widely established as an inhibitor of cytokine signaling, in part through its role in Cullin5-dependent E3 ubiquitin ligase complexes $^{14}$. Xpo5 is an exportin that exports pre-miRNAs and tRNA from the nucleus to the cytoplasm ${ }^{15}$; and Gtpbp8 is a putative guanine nucleotide binding protein of unknown physiological function that is thought to reside in mitochondria ${ }^{16}$. Our data indicate that sgRNA-mediated depletion of each of these proteins indirectly inhibits mitochondrial OXPHOS function, although the exact pathways and mechanisms remain to be understood.

\section{mTOR activity is hyperactivated in OGT-deficient mESCs}

As noted above, Metascape analysis of the protein-protein interaction network identified mTOR signaling, which further showed an interaction connection to mitochondrial function (Fig. 1f). Gene ontology analysis of the 115 candidate genes identified in our sgRNA screen highlighted six genes related to positive regulation of mTOR signaling (Lamtor2, Lamtor3 and Lamtor4, Rraga, Wdr24 and Mios) (Fig. 3a). Lamtor2, 3 and 4 are subunits of the Ragulator complex, which controls the activity of the Rag complex, a heterodimer of two guanine nucleotide-binding proteins, RagA (encoded by Rraga) and RagC (or alternately, RagB and RagD) ${ }^{9}$. Wdr24 and Mios are subunits of the GATOR2 complex, which indirectly senses amino acid levels and activates mTORC1 by inhibiting the GAP activity of GATOR $1^{5,6,9}$. Since mTOR is an established regulator of mitochondrial function at both the transcriptional and translational level ${ }^{7,8}$, we postulated that the increased oxygen consumption rate of Ogt iKO mESCs was driven by increased mTOR activity. In fact, the ATPcompetitive pan-mTOR inhibitor Torin2 restored the high oxygen consumption rate of Ogt iKO mESCs to 
nearly normal levels (Fig. 3b). Moreover, analysis of mTOR activity at a single cell level by flow cytometry and immunostaining showed that the levels of phosphorylation of S6 ribosomal protein on serine 235/236, a reliable marker for mTOR activity, were strikingly increased in Ogt iKO mESCs without a change in total S6 ribosomal protein levels, and this increase was prevented by the mTOR inhibitor Torin2 (Fig. 3c, d; Supplementary Fig.5a).

Since mTOR signaling was strongly up-regulated upon Ogt deletion, we tested whether mTOR inhibition could rescue the viability of OGT-deficient mESCs. Treatment with the mTOR inhibitor Torin2, the mTORC1 inhibitor rapamycin or the AKT inhibitor AKTVIII partially rescued colony morphology and the decrease in mESC cell numbers observed in Ogt iKO cells (Fig. 3e, f; Supplementary Fig. 5b), despite the fact that Ogt fl cells showed a slight decrease in numbers upon treatment with these inhibitors. Similarly, CRISPR-Cas9 mediated disruption of the genes encoding mTOR pathway components Lamtor2, Lamtor4, Rraga and Wdr59 partially rescued cell numbers in Ogt-deleted cells, despite reducing cell numbers somewhat in Ogt fl mESCs (Fig. 3g; Supplementary Fig. 5c, d). Consistent with this observation, expression of sgRNA targeting Lamtor2 inhibited hyperactivated mTOR signaling in Ogt iKO mESCs as judged by immunostaining for phosphorylation of S6 ribosomal protein (Supplementary Fig. 5e). These results point to a functional link between OGT, mTOR activity and mitochondrial function that is essential for the proliferation and survival of OGT-deficient mESC.

\section{Extensive changes in global signaling and protein abundances in Ogt iKO mESC}

To evaluate the global effects of mTOR activation in Ogt-deficient cells, we performed quantitative proteomic and phosphoproteomic analysis of Ogt $\mathrm{fl}$ and Ogt iKO mESCs in the presence and absence of the mTOR inhibitor Torin 2. Phosphoproteomics revealed extensive changes in signaling in mESCs lacking OGT (Fig. 4a). Assessment of kinase activity by post-translational modification-set enrichment analysis (PTM-SEA $)^{17}$ showed significant upregulation of mTOR, RPS6KB1 (S6 kinase) and PKRD1 signaling pathways in Ogtdeficient cells compared to Ogt fl cells (Fig. 4b). As a comparison, AMPKA1 (PRKAA1) kinase, which senses ATP levels in the cell, showed only minor activation upon Ogt deletion. Kinases associated with cell cycle progression, CDK1/2 and CSNK2A1 had diminished activity in Ogt-deficient mESCs (Fig. 4b). Comparing changes in phosphorylation levels with changes in protein levels to assess phospho-site stoichiometry of canonical mTOR signaling components, we found that activating phosphorylations on mTOR were significantly upregulated upon Ogt deletion without changes in mTOR protein levels (Fig. 4c). TSC2, a negative regulator of mTORC1 signaling, showed significant upregulation of its inhibitory phosphorylation site S981, also without changes in protein levels. Ribosomal protein S6 (RPS6), a well-established target of S6K, showed a small though significant decrease in protein levels with a concomitant increase in phosphorylation levels at S235 and S236, indicating an increase in stoichiometry (Fig. 4c). Administration of Torin 2 in Ogt iKO cells decreased the abundance of $20 \%$ of the phosphopeptides dysregulated by Ogt deletion and subsequent mTOR activation (Fig. 4a). Amongst these were several phosphorylation sites downstream of mTOR, including RPS6KB1, RPS6 and EIF4EBP1 (Fig. 4d). Torin2 did not alter a phosphorylation site on mTOR responsive to amino acids, S1261, suggesting mTOR may be sensing changes in amino acid levels ${ }^{18}$. The proteome of Ogt iKO mESCs also showed major changes in protein levels (3,836 of the 8,470 measured) compared to Ogt $f l$ cells, where OGT and OGA were among the most downregulated proteins upon Ogt deletion (Fig. 4e). Gene set enrichment analysis of the proteome-level data also showed increases in proteins involved in PI3K-AKT-mTOR signaling in Ogt-deficient mESCs (Fig. 4f). Together these data show indicate Ogt deletion leads to activation of mTOR/S6K signaling, and extensive remodeling of the proteome and phosphoproteome, which provide a resource for the future study of crosstalk between O-GICNAc modification and phosphorylation.

\section{OGT deficiency promotes mTOR translocation by increasing proteasome activity}


We investigated the mechanisms underlying aberrant mTOR activation in the absence of OGT. Notably, the six hits from our CRISPR/Cas9 screen were related to mTOR translocation to the lysosomal membrane mediated by the Rag and Ragulator complexes (Rraga, Lamtor 2-4), and to amino acid sensing mediated by the GATOR2 complex (Wdr24 and Mios) ${ }^{5,6}$. Moreover, an amino acid-responsive phosphorylation site on mTOR was induced upon Ogt deletion and was not altered by Torin2 (Fig. 4d). We therefore assessed mTOR subcellular localization by immunocytochemistry of Ogt $\mathrm{fl}$ and Ogt iKO mESCs, and also monitored steadystate amino acid levels in these cells (Fig. 5a, b). In Ogt fl mESCs, mTOR staining was diffusely localized to the cytoplasm, with little overlap with the lysosomal marker LAMP1, whereas in Ogt iKO mESCs, mTOR showed perinuclear staining that overlapped considerably with LAMP1 (Fig. 5a; Supplementary Fig. 6a). sgRNA-mediated disruption of Lamtor2 inhibited mTOR translocation to the lysosome in Ogt iKO mESCs (Supplementary Fig. 6b). These data confirmed that mTOR was activated by translocation to the lysosome in the absence of OGT.

The lysosomal translocation and activation of mTOR correlated with a global increase in amino acid levels in Ogt iKO mESCs, apparent as early as 4 days or 6 days after 4-OHT treatment (Fig. 5b). Steady-state amino acid levels are controlled by the balance between protein synthesis, which depends on mRNA translation by ribosomes, and protein degradation, effected by proteasomes, lysosomes and autophagosomes. We focused on 26S proteasome activity, which was previously reported to be reversibly inhibited by OGT through direct $O$ GlcNAc modification of proteasome subunits including the ATPase Rpt2 (also known as Psmc1) ${ }^{19}$. Indeed, proteasome activity was significantly increased in Ogt iKO compared to Ogt floxed mESCs, this increase in activity was completely inhibited by treatment with the proteasome inhibitor MG132 (Fig. 5c). Moreover, treatment with $10 \mu \mathrm{M}$ MG132 for 2.5 hours significantly reduced the increase in mTOR activity caused by Ogt deletion as assessed by flow cytometry or immunostaining for phospho-S6, while the levels of total S6 ribosomal protein remained unchanged (Fig. 5d, e; Supplementary Fig. 6c). MG132 treatment under these conditions also significantly reduced the lysosomal localization of mTOR in Ogt iKO mESC, as indicated by the poor overlap of mTOR staining with the lysosome marker LAMP1 (Fig. 5f; Supplementary Fig. 6d), and reduced the aberrant increase of amino acids in Ogt iKO mESCs (Fig. $\mathbf{5 g}$ ).

In addition to the direct O-GIcNAc modification of proteasome subunit Psmc1 ${ }^{19}$, Ubiquilin-2, ubiquitin associated proteins Ubap2 (Ubiquitin-associated protein 2) and Ubap2l (Ubiquitin-associated protein 2-like) have been reported to be directly O-GlcNAcylated (Supplementary Table 1) ${ }^{20,21}$. Ubiquilin-2 is a connector between the proteasome and the polyubiquitin chains on targeted proteins through its $\mathrm{N}$-terminal ubiquitin-like domain (UBL) and C-terminal ubiquitin-associated domain (UBA) ${ }^{22}$. Both Ubap2 and Ubap2I contain UBA domains and play important roles in the ubiquitin-proteasome pathway ${ }^{23,24}$. To corroborate these findings in mESCs, we precipitated whole cell lysates of Ogt $\mathrm{fl}$ mESCs with wheat germ agglutinin (WGA), a lectin that recognizes the O-GIcNAc modification, and immunoblotted for Pmsc1, Ubiquilin-2, Ubap2 and Ubap2l. These experiments confirmed previous findings (Supplementary Table 1) ${ }^{21}$ showing that Pmsc1/Rpt2, Ubiquilin-2, Ubap2 and Ubap2I were O-GlcNAc-modified in Ogt fl mESCs (Fig. 5h). In the case of Ubiquilin-2, we observed clear enrichment for a slowly-migrating, presumably $O$-GlcNAc-modified protein species. Together these data suggest that proteasomal subunit and ubiquitin associated proteins are O-GlcNAc modified by Ogt and in the absence of OGT, high proteasome activity and consequent high intracellular amino acid levels result in aberrant mTOR activation and increased mitochondrial OXPHOS, thus causing decreased proliferation and decreased viability of mESCs.

\section{Ogt-deficient T cells also show increased amino acid levels and increased mTOR activation}

Next, we asked if what we observed in mESCs is a general mechanism across mammalian cell types. To answer this question, we used $\mathrm{CD}^{+} \mathrm{T}$ cells isolated from male Ogf ${ }^{\text {floxed }} \mathrm{Cre}^{-E R T 2^{+/ K l}}$ Rosa26-LSL-YFP $^{+/ K I}$ mice. At day 6 after 4-OHT treatment, the O-GlcNAc modification was completely eliminated in Ogt iKO CD8 ${ }^{+}$ 
T cells as assessed by flow cytometry (Supplementary Fig. 7a). As expected, Ogt iKO CD8 ${ }^{+} \mathrm{T}_{\text {cells showed a }}$ significant impairment of growth rate (Supplementary Fig. 7b). Consistent with our results in mESCs, Ogtdeficiency in $\mathrm{CD}^{+} \mathrm{T}$ cells led to a significant increase in amino acid levels (Supplementary Fig. 7c) and mTOR activation (Supplementary Fig7d, e). The levels of phosphorylation of S6 ribosomal protein on serine 235/236 were significantly increased in Ogt iKO CD8 ${ }^{+}$T cells while the levels of total S6 ribosomal protein slightly decreased analyzed by flow cytometry (Supplementary Fig. 7d, e). Treatment with the mTOR inhibitor Torin2 and mitochondrial inhibitor Rotenone partially rescued the decrease of cell numbers in Ogt iKO CD8 ${ }^{+} \mathrm{T}$ cells, despite a decrease of cell numbers in Ogt $\mathrm{fl} \mathrm{CD8}{ }^{+} \mathrm{T}$ cells upon treatment with these inhibitors (Supplementary Fig. 7f, g). Together, these data suggested that Ogt controls cell viability through the proteasome/ mTOR/mitochondrial axis in both embryonic stem cells and somatic cells.

\section{Discussion}

Unlike other post-translational modifications such as phosphorylation that are regulated by hundreds of kinases and phosphatases, the O-GIcNAc modification is catalyzed uniquely by OGT and OGA, enzymes that add and remove $O$-GlcNAc from cellular proteins, respectively ${ }^{25}$. As the only enzyme that modifies the hydroxyl groups of serine and threonine residues on intracellular proteins with GlcNAc, OGT is highly conserved across species $^{1,25}$ and is absolutely required for the survival of mammalian cells including mESCs, mouse embryonic fibroblasts (MEFs) and mouse $\mathrm{CD}^{+} \mathrm{T}$ cells ${ }^{3,4,26}$. However, why OGT is essential for mammalian cell viability remains unclear ${ }^{1}$. Our genome-wide CRISPR/Cas9 screen, performed in mESCs subjected to inducible deletion of Ogt, provides important insights, revealing that mitochondrial dysfunction is a major cause for the block in cell proliferation and eventual loss of cell viability induced by OGT deficiency. We have traced this phenotype to mTOR hyperactivity, resulting from increased steady-state amino acid levels and secondary to increased proteasomal function (Supplementary Fig. 8). This mechanism operates in both mESCs and CD8 ${ }^{+}$ T cells. A useful product of the screen is that we were able to obtain viable, proliferating Ogt-deficient cells by inhibiting mTOR hyperactivation and the aberrant increase in mitochondrial oxidative phosphorylation. To our knowledge, this is the first time that Ogt deficiency has been achieved while maintaining cell proliferation. The availability of such inducibly Ogt-deficient cells will greatly facilitate our understanding of the many intracellular roles of OGT.

Our study connects OGT deficiency directly to overactivity of the mitochondrial electron transport chain. Ogt-deficient mESCs displayed an increased level of mitochondrial OXPHOS. Despite its toxicity to normal cells, the mitochondrial complex 1 inhibitor rotenone partially rescued the proliferation defect of Ogt-deficient cells. Depletion of Socs3 and Xpo5, two proteins not obviously associated with mitochondrial function, and Gtpbp8, a protein annotated as mitochondrial but of unknown function, returned mitochondrial OXPHOS to almost normal levels while also rescuing the proliferation defect of Ogt-deficient cells. Notably, rescue by the top sgRNA candidates identified in our whole-genome screen was incomplete; given that OGT has thousands of substrates, other mechanisms (in addition to mTOR hyperactivation and the associated mitochondrial dysfunction) might contribute to the defect in cell proliferation in Ogt-deficient cells.

mTORC1 is a bonafide nutrient sensor: it regulates mRNA translation, metabolism and protein turnover in response to intracellular and extracellular signals such as nutrients and growth factors ${ }^{5,27}$. mTOR activation led to increased levels of OGT and O-GIcNAc modification ${ }^{28}$; likewise, pharmacological inhibition of mTOR led to decreased OGT expression concomitant with a global reduction of O-GlcNAc modification ${ }^{29}$. Therefore, OGT activity is enhanced by PI3K-AKT-mTOR signaling, while conversely, OGT inhibits mTOR translocation and activation in a negative feedback loop that maintains the proper balance of proteasome and mTOR activity to regulate mitochondrial fitness, a parameter essential for cell viability. Disruption of this balance in Ogt-deficient cells induces proteasome and mTOR hyperactivation, with subsequent deleterious effects on mitochondrial function and a dramatic block in cell proliferation, leading eventually to a decrease in cell viability. 
Aberrant metabolism is one of the hallmarks of cancer, and many of the proteins that play important roles in the proteasome/ mTOR/ mitochondrial axis (Supplementary Fig. 8) are aberrantly expressed in cancer. Increased levels of OGT expression and O-GIcNAc modification are often observed in cancer cells ${ }^{30-32}$. Hyperactivation of mTOR signaling is observed in many types of cancers; mTOR signaling is the second most frequently altered signaling pathway in cancer; and multiple mTOR inhibitors have been used in cancer clinical trials $^{33,34}$. Increased expression of proteasome subunits and high proteasome activity are also characteristic of many cancer types ${ }^{35-37}$, and proteasome inhibitors have been used in the clinic for the treatment of hematological malignancies ${ }^{38}$. Several proteasome subunits and ubiquitin-associated proteins, including Psmc1, Ubap2, Ubap2l and Ubiquilin2, are targets for O-GlcNAcylation by OGT and display aberrant expression in cancer cells ${ }^{39-44}$. Therefore, a detailed investigation of OGT activity in proteasome/ mTOR signaling in different cancer cell types may provide novel targets for cancer therapy in the future. 


\section{Acknowledgements}

We thank Dr. Joyce Chen and Dr. Anand Balasubramani for generating Ogftoxed Cre-ERT2 $2^{K / K l}$ mice and Ogftoxed Rosa26-LSL-YFP ${ }^{K I K l}$ mice; Dr. Mohit Jain (UCSD) and members of the Rao laboratory for suggestions and discussions. We thank Dr. Natasha Zachara from Johns Hopkins University School of Medicine for providing Ogt floxed mice. We thank Dr. Ronald M. Evans (Salk Institute) for providing wildtype OGT plasmid. We thank C. Kim, D. Hinz, C. Dillingham, M. Haynes and S. Ellis at the La Jolla Institute Flow Cytometry facility for help with cell sorting experiments; J. Day, S. Alarcon, H. Dose, K. Tanguay and A. Hernandez of the La Jolla Institute Sequencing facility for help with next-generation sequencing. We thank Olga Zagnitko at Cancer Metabolism Core at Sanford Burnham Prebys Medical Discovery Institute for assistance with GC/MS analyses. This work was supported by National Institutes of Health (NIH) grants R35 CA210043 and R01 Al109842 (to A.R.). FACSAria II Cell Sorter was acquired through the Shared Instrumentation Grant (SIG) Program S10 RR027366 and Hiseq 2500 was funded by S10OD016262. The Sanford Burnham Prebys Cancer Metabolism Core is supported by NCl Cancer Center Support Grant P30 CA030199. X.L. was supported by a postdoctoral Fellowship from CIRM UCSD Interdisciplinary Stem Cell Research \& Training Grant II (TG2-01154). H.S. was supported by Pew Latin American Fellows Program from the Pew Charitable Trusts.

\section{Author contributions}

A.R. and X.L. conceived the project. X.L. and X.Y. performed experiments, analyzed and interpreted the results. X.L. prepared samples for seahorse, GC/MS, proteomic and phosphoproteomic experiments. H.S. assisted with experiments. D.A.S. performed the seahorse experiments and GC/MS profiling of amino acids. R.A.B and S.A.M. performed the proteomic and phosphoproteomic experiments, analyzed and interpreted the data. S.A.C supervised the proteomic and phosphoproteomic experiments. A.R. supervised project planning and execution. X.L., X.Y., S.A.M. and A.R. wrote the manuscript, and all authors proofread the manuscript and provided editorial input.

\section{Competing interests}

None of the authors has any competing interests. 


\section{Figure Legends}

\section{Fig. 1 | Genome-wide CRISPR-Cas9 screen to identify key regulators of cell survival in OGT-deficient} mESCs.

a, Phase contrast images of Ogt fl mESCs treated with or without 4-OHT for 8 days. b, Cumulative growth curves of Ogt $\mathrm{fl}$ mESCs treated with or without 4-OHT at day 0 and counted at each passage (every 3 days) thereafter until day 9. Data are shown as mean $\pm \mathrm{SD}(\mathrm{N}=3)$. c, Strategy for the genome-wide CRISPR-Cas9 viability screen to identify key regulators of cell growth in OGT-deficient mESCs. d, Rank of enriched hits by gene score, calculated based on the log fold changes of all sgRNAs targeting each gene and the number of sgRNAs targeting each gene that reached statistical significance. 115 enriched hits in both biological replicates are shown in purple. A few of the most highly scored hits (Hmbs, Gtpbp8, Opa1, Socs3 and Mrp/50) are labeled. e, Gene ontology analysis of biological pathways of the 115 enriched hits. $\mathbf{f}$, Metascape visualization of the protein-protein interactome network of the 115 highly-scored hits from the CRISPR screen. Each MCODE complex identified by Metascape was assigned a unique color. g, Three MCODE complexes and their associated functional pathways identified by Metascape.

\section{Fig. 2 | OGT deficiency results in mitochondrial dysfunction}

a, Analysis of oxygen consumption rate (OCR) using Seahorse XFe24 in Ogt fl and Ogt iKO mESCs treated without or with 4-OHTrespectively for 6 days. Data are shown as mean $\pm S D(N=3)$. $b$, Relative cell numbers of Ogt $f l$ and Ogt iKO mESCs treated without or with 4-OHT respectively and with or without the mitochondrial Complex I inhibitor rotenone $(75 \mathrm{nM})$ for 8 days. Data are shown as mean $\pm \mathrm{SD}(\mathrm{N}=3)$. c, Phase contrast images of Ogt $\mathrm{fl}$ and Ogt iKO mESCs expressing non-targeting sgRNA (sgNT) or sgRNAs targeting the indicated genes (Socs3, Xpo5, Opa1 and Gtpbp8) and treated without or with 4-OHT respectively for 8 days. d, Relative cell numbers of Ogt $\mathrm{fl}$ and Ogt iKO mESCs expressing non-targeting sgRNA (sgNT) or sgRNAs targeting the indicated genes (Socs3, Xpo5, Opa1, Gtpbp8, Mrpl50, Ythdc1, Uros, Hmbs, Tet2 or Tet1) and treated without or with 4-OHT respectively for 8 days. Data are shown as mean $\pm \mathrm{SD}(\mathrm{N}=3)$. e-g, Analysis of oxygen consumption rate (OCR) using Seahorse XFe24 in Ogt fl and Ogt iKO mESCs expressing sgRNAs targeting Gtpbp8 (e), Socs3 (f) and Xpo5 $(\mathbf{g})$ and treated without or with 4-OHT respectively for 6 days. Data are shown as mean $\pm \mathrm{SD}(\mathrm{N}=3)$.

\section{Fig. 3 | mTOR is hyperactivated in OGT-deficient mESCs}

a, Rank of enriched genes by gene score. Genes related to positive regulation of mTOR signaling were highlighted. b, Analysis of oxygen consumption rate (OCR) using Seahorse XFe24 in Ogt fl and Ogt iKO mESCs treated without or with 4-OHT respectively and with or without Torin2 (25 nM) for 6 days. Data are shown as mean $\pm \mathrm{SD}(\mathrm{N}=3)$. c, Immunohistochemistry of Ogt fl and Ogt iKO mESCs treated without or with 4$\mathrm{OHT}$ respectively for 6 days. Cells were stained with antibody against phospho-S6 ribosomal protein. Nucleus staining: DAPI (blue). Scale bar: $10 \mu \mathrm{m}$. d, Flow cytometry analysis of phospho-S6 (left panel) and total S6 (right panel) ribosomal protein in Ogt $\mathrm{fl}$ and Ogt iKO mESCs treated without or with 4-OHT respectively for 6 days and then with or without $200 \mathrm{nM}$ Torin2 for 2 hours. Data are representative of three biological replicates. e, Left, Simplified schematic diagram of mTOR pathway activation and the proteins targeted by the inhibitors Torin2 $(25 \mathrm{nM})$, rapamycin $(50 \mathrm{nM})$ and AktVIII $(7.5 \mu \mathrm{M})$. Right, Phase contrast images of Ogt fl and Ogt iKO mESCs treated with or without Torin2. f, Relative cell numbers of Ogt fl and Ogt iKO mESCs treated without or with 4-OHT respectively and with or without Torin2 (25 nM), Rapamycin (50 nM) and AktVIII (7.5 $\mu \mathrm{M})$ for 8 days. Data are shown as mean $\pm \mathrm{SD}(\mathrm{N}=3)$. g, Relative cell numbers of Ogt $\mathrm{fl}$ and Ogt iKO mESCs expressing gRNAs against the indicated genes (Lamtor2, Lamtor4, Raga and Wdr59) and treated without or with 4-OHT respectively for 8 days. Data are shown as mean $\pm S D(N=3)$. 


\section{Fig. 4 | Extensive changes in global signaling and protein abundances in Ogt iKO mESC.}

a, K-means clustering analysis of all regulated (B-H corrected adjusted $\mathrm{p}$-value $\geq 0.05)$ phosphopeptides as determined by a moderated F-test. Example phosphopeptides for canonical mTOR pathway components are highlighted.Italicized phosphosite numbers indicate phosphosite assignment ambiguity. b, Normalized enrichment scores for kinase activity as determined by Post-translational modification-set enrichment analysis (PTM-SEA) of phospho-proteomics data performed in Ogt fl and Ogt iKO mESCs. Top three pathways in both directions, as well as their respective p-values are shown. PRKAA1 (AMPKA1) is included as a comparison. Only mouse-derived PTM sets were used for the analysis. c, Changes in phosphopeptide and protein levels of mTOR signaling components in Ogt $\mathrm{fl}$ and Ogt iKO mESCs as determined by quantitative proteomics. Grey regions indicate non-statistically significant changes in the respective datasets. Italicized phosphosite numbers indicate phosphosite assignment ambiguity. d, Changes in phosphorylation levels on mTOR signaling components upon Ogt deletion alone (y-axis) and Ogt deletion in the presence of Torin2 (x-axis). Both are relative to Ogt $\mathrm{fl}$. Italicized phosphosite numbers indicate phosphosite assignment ambiguity. "Iso 2" refers to isoform 2 of RPS6KB1. e, Volcano plot of differentially abundant proteins between Ogt floxed and Ogt iKO mESCs. B-H corrected $p$ values of less than or equal to 0.05 are highlighted. $\mathbf{f}$, Leading edge analysis for proteome level-GSEA of proteins involved in the PI3K-AKT-MTOR pathway. Positive enrichment indicates up in Ogt iKO; negative, down in Ogt iKO. Normalized enrichment score and false discovery reate (FDR, q-value) are shown.

\section{Fig. 5 | OGT deficiency promotes the translocation of mTOR by increasing proteasome activity.}

a, Immunohistochemistry of Ogt $f l$ and Ogt iKO mESCs treated without or with 4-OHT respectively for 6 days. Cells were stained with antibodies against mTOR and Lamp1. Nucleus staining: DAPI (blue). Scale bar: 10 $\mu \mathrm{m}$. b, Relative amino acid levels in Ogt $\mathrm{fl}$ and Ogt iKO mESCs treated without or with 4-OHT respectively for 4 or 6 days. Data are shown as mean $\pm \mathrm{SD}(\mathrm{N}=3)$. c, Chymotrypsin-like proteasome activity in Ogt $\mathrm{fl}$ and Ogt iKO mESCs treated without or with 4-OHT respectively for 4 or 6 days. Data are shown as mean $\pm S D(N=3)$. d, Flow cytometry analysis of phospho-S6 (left panel) and total S6 (right panel) ribosomal protein in Ogt fl and Ogt iKO mESCs treated without or with 4-OHT respectively for 6 days and then with or without $10 \mu \mathrm{M}$ MG132 or $200 \mathrm{nM}$ Torin2 for 2.5 hours. e, Relative mean fluorescence intensity (MFI) of phospho-S6 and S6 ribosomal protein in three independent experiments similar to that shown in (d). f, Immunohistochemistry of Ogt $\mathrm{fl}$ and Ogt iKO mESCs treated without or with 4-OHT respectively for 6 days and then with or without $10 \mu \mathrm{M}$ MG132 for 2.5 hours. Cells were stained with antibodies against mTOR and Lamp1. Nucleus staining: DAPI (blue). Scale bar: $10 \mu \mathrm{m} . \mathbf{g}$, Relative amino acid levels in Ogt $\mathrm{fl}$ and Ogt iKO mESCs treated without or with 4-OHT respectively for 4 days and then with or without $10 \mu \mathrm{M}$ MG132 for 2.5 hours. $\mathbf{h}$, Western blot of UBAP2, UBAP2L, Ubiquilin 2 and PSMC1 proteins after WGA pull down of cell lysates from Ogt $f / \mathrm{mESCs}$. FT: Flow Through. 


\section{Materials and Methods}

\section{Mice}

Ogt floxed mice deposited at Jackson Laboratory by the J. Marth laboratory ${ }^{3,4}$ and backcrossed to C57BL/6 mice for 12 generations were kindly provided by Dr. Natasha Zachara (Johns Hopkins). B6.129-

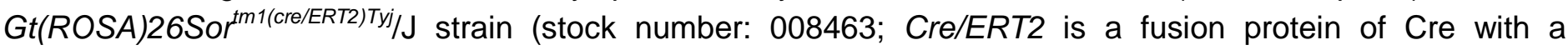
modified estrogen receptor, which enters the nucleus only after it binds 4-hydroxytamoxifen) and B6.129X1-

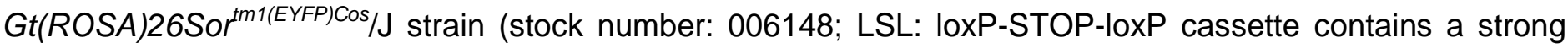
transcriptional STOP site flanked by loxP sites, which turns on EYFP expression upon Cre-mediated deletion of loxP sites) were purchased from the Jackson Laboratory. All mice were on the B6 background and maintained in a specific pathogen-free animal facility in the La Jolla Institute for Immunology. All animal procedures were reviewed and approved by the Institutional Animal Care and Use Committee of the La Jolla Institute for Immunology and were conducted in accordance with institutional guidelines.

\section{Generation and culture of mouse embryonic stem cells}

Ogt ${ }^{\text {floxed }}$ Cre-ERT2 $2^{K I / K I}$ mice were crossed with Ogf floxed Rosa26-YFP ${ }^{K I / K I}$ mice to obtain Ogt floxed $C r e-E R T 2^{+/ K I}$ Rosa26-LSL-YFP ${ }^{+/ K l}$ blastocysts (embryonic day 3.5) to generate male and female Ogt floxed mESC lines. Ogt deletion was then induced by the addition of $1 \mu \mathrm{M}$ 4-hydroxytamoxifen (4-OHT) (TOCRIS, catalog number: 3412). Mouse ESCs were maintained on mitomycin C-treated mouse embryonic fibroblasts (MEFs; feeder cells) with LIF in Knockout DMEM medium (ThermoFisher Scientific, catalog number: 10829018) supplemented with 15\% KOSR (KnockOut Serum Replacement, ThermoFisher Scientific, catalog number: 10828028), 2 mM L-Glutamine, 1 X MEM Non-Essential Amino Acids, $50 \mu \mathrm{M} \beta$-Mercaptoethanol. Cell numbers were counted by flow cytometry on a BD Accuri C6 (BD Biosciences).

\section{Plasmids}

Wildtype OGT plasmid was kindly provided by Dr. Ronald M. Evans (Salk Institute). Wildtype OGT was subcloned into a lentiviral vector pLV-EF1a-IRES-Puro (Addgene \#85132).

\section{Lentiviral packaging and transduction}

Lentiviral packaging was performed in HEK293T cells using Lipofectamine 2000 (ThermoFisher Scientific) according to the manufacturer's protocol. Briefly, pCMV-VSVG (Addgene, \#8454), psPAX2 (Addgene, \#12260) and lentiGuide plasmids were transfected at the ratio of 1:2:2. Lentiviral supernatant was harvested at $48 \mathrm{hr}$ post-transfection and used for mESC infection.

\section{CRISPR/Cas9 Screen}

An Ogt ${ }^{\text {floxed }}$ Cre-ERT2 ${ }^{+/ K I}$ Rosa26-LSL-YFP ${ }^{+/ K I}$ mESC cell line stably expressing Cas9 was generated by lentiviral transduction of the parental cells with lentiCas9-Blast (Addgene \#52962). CRISPR sgRNA library lentiGuide-Puro-Brie (Addgene, \#73633), which contains a pool of 78,637 sgRNAs targeting 19,647 genes was transduced into mESCs with $\mathrm{MOI}<0.3$ and an average of $400 x$ coverage. After puromycin selection for 7 days to obtain cells stably expressing the sgRNAs, the cells were split into two groups, an untreated (Ogt fl) group that served as the control, and a second group treated with 4-OHT for 6 days to induce Ogt deletion (Ogt iKO). On day 13, YFP-negative cells from the control Ogt fl group and $\mathrm{YFP}^{+}$cells from the Ogt iKO group were sorted and used for library preparation for next generation deep sequencing. 


\section{Library Preparation, sequencing and data analysis}

Genomic DNA was isolated using Quick-gDNA MidiPrep kit (Zymo Research, D3100) according to the manufacturer's protocol. Library preparation was performed as described in (Feng Zhang, 2017, Nat Prot). sgRNA inserts were PCR-amplified using NEBNext High Fidelity PCR Master Mix (NEB, M0541). The resulting PCR amplicons were purified using Ampure XP Beads and sequenced using the HiSeq 2500 system (Illumina) to assess changes in abundance of sgRNAs between Ogt $f l$ and Ogt iKO groups. The sequencing data were analyzed using the PinAPL-Py platform ${ }^{12}$.

\section{Seahorse Mito Stress assay and amino acid measurement}

A Seahorse XFe24 Bioanalyzer (Agilent) was used to determine OCR (oxygen consumption rate). 100,000 mESCs per well were seeded in a Seahorse XFe24 cell culture plate precoated with Matrigel Matrix (Corning) one day prior to the assay. OCR was measured at baseline and after sequential addition of (i) oligomycin (2 $\mu \mathrm{M}$; an ATP synthase inhibitor); (ii) carbonyl cyanide-4-(trifluoromethoxy) phenylhydrazone (FCCP) (1 $\mu \mathrm{M}$; a mitochondrial uncoupler); (iii) rotenone (1 $\mathrm{MM}$; a complex I inhibitor) and antimycin A (1 $\mu \mathrm{M}$; a complex III inhibitor).

\section{Amino acid measurement}

The samples were collected in 50\% methanol with $20 \mu \mathrm{M} \mathrm{L-Norvaline.} \mathrm{The} \mathrm{levels} \mathrm{of} \mathrm{amino} \mathrm{acids} \mathrm{were} \mathrm{profiled}$ by gas chromatography/mass spectrometry (GC/MS) at Cancer Metabolism Core, Sanford Burnham Prebys Medical Discovery Institute using methods described in reference ${ }^{45}$. Arginine is unstable in this analytical procedure and is converted to ornithine.

\section{Proteasome activity measurement}

The chymotrypsin-like protease activity associated with the proteasome complex was measured using the Proteasome-Glo Cell-Based Assay Kit (G8660, Promega) as described by the manufacturer. Briefly, mESCs were trypsinized and washed with culture medium, 100,000 cells per well were seeded into a 96-well plate and incubated with or without $10 \mu \mathrm{M} \mathrm{MG} 132$ for $90 \mathrm{~min}$ at $37^{\circ} \mathrm{C}, 5 \% \mathrm{CO}_{2}$. The plate was then allowed to equilibrate to room temperature for $15 \mathrm{~min}$ before the addition of $100 \mu \mathrm{L}$ proteasome Glo reagent containing the bioluminescent substrate Suc-LLVY amino-luciferin, which releases amino-luciferin upon cleavage by the proteasome. The luminescent signals produced upon cleavage were measured using a SpectraMax Luminometer.

\section{Quantitative real-time PCR (qRT-PCR)}

Quantitative real-time PCR was performed using Universal SYBR Green Master Mix (Roche) and analyzed using a Step One Plus real-time PCR system (Applied Biosystems) according to the manufacturer's instructions and the data were normalized for Gapdh expression. The primers used for qRT-PCR are listed in

\section{Supplementary Table 2.}

\section{Immunohistochemistry}

Immunohistochemistry was performed as described previously ${ }^{46}$ with the primary antibodies (Clone name, conjugated fluorescence, dilution, manufacturer and catalog number shown in brackets): anti-O-Linked $\mathrm{N}$ Acetylglucosamine antibody (RL2, Alexa Fluor 647, 1:200, Abcam, ab201994); Phospho-S6 Ribosomal Protein 
(Ser235/236) (D57.2.2E, Alexa Fluor 555, 1:200, Cell Signaling Technology, 39855); S6 Ribosomal Protein (54D2, Alexa Fluor 647; 1:200, Cell Signaling Technology, 55485); Lamp1 (1D4B, unconjugated, 1:50, Developmental Studies Hybridoma Bank, 1D4B); mTOR (7C10, unconjugated, 1:200, Cell Signaling Technology, 2983S). The colocalization correlation coefficients (Pearson's correlation) between mTOR and Lamp1 were analyzed using software ImageJ2 with the plugin Coloc 2.

\section{Flow cytometry}

mES cells were trypsinized into single cells and used for staining or cell sorting. The antibodies used for the staining are as following (Clone name, conjugated fluorescence, dilution, manufacturer and catalog number shown in brackets): Phospho-S6 Ribosomal Protein (Ser235/236) (D57.2.2E, Alexa Fluor 555, 1:200, Cell Signaling, 39855); S6 Ribosomal Protein (54D2, Alexa Fluor 647; 1:200, Cell Signaling, 55485); anti-O-Linked N-Acetylglucosamine antibody (RL2, Alexa Fluor 647, 1:200, Abcam, ab201994).

\section{Pulldown of O-GIcNAcylated proteins in cell lysates with wheatgerm agglutinin (WGA)}

WGA pull down was performed using Pierce ${ }^{\text {TM }}$ Glycoprotein Isolation Kit, WGA (Thermo Scientific) as described in the manufacturer's protocol. Briefly, $800 \mu \mathrm{g}$ of total cell lysates prepared with RIPA buffer were added to $200 \mu \mathrm{l}$ of WGA lectin resin in a total volume of $500 \mu \mathrm{l}$ bind buffer and incubated with mixing for 20 minutes at room temperature. The beads were washed four times in wash buffer; O-GlcNAcylated proteins were eluted from the resin at $95^{\circ} \mathrm{C}$, and the resulting eluent and the first flow-through from the column were analyzed by Western blotting with antibodies against PSMC1 (Proteintech, 11196-1-AP), Ubiquilin 2 (Novus Biologicals, H00029978-M03), UBAP2 (Bethyl Laboratories Inc, A304-627A), UBAP2L (Bethyl Laboratories Inc, A300-533A).

\section{Proteomics and Phosphoproteomics}

mESC pellets were pelleted, snap-frozen with liquid nitrogen, and stored at $-80^{\circ} \mathrm{C}$ until prepared for mass spectrometric analysis. Cell pellets were processed and analyzed as previously described with minor modifications ${ }^{47,48}$. Samples were analyzed on a $Q$ Exactive HF-X quadrupole-Orbitrap mass spectrometer (Thermo Fisher Scientific).

Mass spectrometric data was analyzed using Spectrum Mill (Aglient) searching a Uniprot mouse database (12/28/2017) with 47,069 entries including common laboratory contaminants. All phosphosite assignments with less than $99 \%$ localization confidence are italicized and reported by Spectrum Mill without modification. TMT 11 ratios were generated using the median of all channels. Biological replicates with a Pearson's $r$ correlation of less than 0.2 were excluded. This was applied to one sample in the phosphoproteomics analysis, which is less stable than proteome changes ${ }^{48}$. The moderated T-test (pairwise comparisons) and moderated F-test (comparisons across all samples) were performed using Limma. GSEA was performed as previously described using only Hallmark gene sets ${ }^{49}$. PTM-SEA was performed as previously described, using only the mouse database $^{17}$.

\section{$\mathrm{CD8}^{+} \mathrm{T}$ cell isolation and activation}

$\mathrm{CD}^{+} \mathrm{T}$ cells were isolated from spleen and lymph nodes of Ogt ${ }^{f l o x e d}$ Cre-ERT2 ${ }^{+/ K I}$ Rosa26-LSL-YFP ${ }^{+/ K I}$ mice using Dynabeads Untouched Mouse CD8 Cells Kit (ThermoFischer, 11417D), and activated with plate-bound anti-CD3 (clone 2C11, BioXcell) and anti-CD28 (clone 37.51, BioXcell) antibodies at $1 \mu \mathrm{g} / \mathrm{ml}$ in the presence of $100 \mathrm{U} / \mathrm{ml}$ recombinant human IL-2 (rhIL-2). 4-OHT (1 $\mu \mathrm{M})$ was added to induce Ogt deletion. 


\section{Statistical Analysis}

All values are shown as means \pm SD. To determine the significance between groups, comparison was made using Student's $t$ test. For all statistical tests, the 0.05 confidence level was considered statistically significant. In all figures, * denotes $p<0.05$ and ${ }^{* *}$ denotes $p<0.01$ in an unpaired Student's t-test.

\section{Data Availability}

The authors declare that all data supporting this study are available within the article, supplementary figures and supplementary tables or from the corresponding authors upon reasonable request. 


\section{References:}

1 Levine, Z. G. \& Walker, S. The Biochemistry of O-GlcNAc Transferase: Which Functions Make It Essential in Mammalian Cells? Annual review of biochemistry 85, 631-657, doi:10.1146/annurevbiochem-060713-035344 (2016).

2 Bond, M. R. \& Hanover, J. A. A little sugar goes a long way: the cell biology of O-GlcNAc. The Journal of cell biology 208, 869-880, doi:10.1083/jcb.201501101 (2015).

3 Shafi, R. et al. The O-GlcNAc transferase gene resides on the $\mathrm{X}$ chromosome and is essential for embryonic stem cell viability and mouse ontogeny. Proceedings of the National Academy of Sciences of the United States of America 97, 5735-5739, doi:10.1073/pnas.100471497 (2000).

O'Donnell, N., Zachara, N. E., Hart, G. W. \& Marth, J. D. Ogt-dependent X-chromosome-linked protein glycosylation is a requisite modification in somatic cell function and embryo viability. Molecular and cellular biology 24, 1680-1690, doi:10.1128/mcb.24.4.1680-1690.2004 (2004).

Condon, K. J. \& Sabatini, D. M. Nutrient regulation of mTORC1 at a glance. Journal of cell science 132 , doi:10.1242/jcs.222570 (2019).

Liu, G. Y. \& Sabatini, D. M. mTOR at the nexus of nutrition, growth, ageing and disease. Nature reviews. Molecular cell biology 21, 183-203, doi:10.1038/s41580-019-0199-y (2020).

Morita, M. et al. mTORC1 controls mitochondrial activity and biogenesis through 4E-BP-dependent translational regulation. Cell metabolism 18, 698-711, doi:10.1016/j.cmet.2013.10.001 (2013).

Cunningham, J. T. et al. mTOR controls mitochondrial oxidative function through a YY1-PGC-1alpha transcriptional complex. Nature 450, 736-740, doi:10.1038/nature06322 (2007).

Sancak, Y. et al. The Rag GTPases bind raptor and mediate amino acid signaling to mTORC1. Science (New York, N.Y.) 320, 1496-1501, doi:10.1126/science.1157535 (2008).

Kim, E., Goraksha-Hicks, P., Li, L., Neufeld, T. P. \& Guan, K. L. Regulation of TORC1 by Rag GTPases in nutrient response. Nature cell biology 10, 935-945, doi:10.1038/ncb1753 (2008).

11 Doench, J. G. et al. Optimized sgRNA design to maximize activity and minimize off-target effects of CRISPR-Cas9. Nature biotechnology 34, 184-191, doi:10.1038/nbt.3437 (2016).

Spahn, P. N. et al. PinAPL-Py: A comprehensive web-application for the analysis of CRISPR/Cas9 screens. Scientific reports 7, 15854, doi:10.1038/s41598-017-16193-9 (2017).

Zhou, Y. et al. Metascape provides a biologist-oriented resource for the analysis of systems-level datasets. Nature communications 10, 1523, doi:10.1038/s41467-019-09234-6 (2019).

14 Babon, J. J., Sabo, J. K., Zhang, J. G., Nicola, N. A. \& Norton, R. S. The SOCS box encodes a hierarchy of affinities for Cullin5: implications for ubiquitin ligase formation and cytokine signalling suppression. Journal of molecular biology 387, 162-174, doi:10.1016/j.jmb.2009.01.024 (2009).

15 Wu, K., He, J., Pu, W. \& Peng, Y. The Role of Exportin-5 in MicroRNA Biogenesis and Cancer. Genomics, proteomics \& bioinformatics 16, 120-126, doi:10.1016/j.gpb.2017.09.004 (2018).

16 Maiti, P., Kim, H. J., Tu, Y. T. \& Barrientos, A. Human GTPBP10 is required for mitoribosome maturation. Nucleic acids research 46, 11423-11437, doi:10.1093/nar/gky938 (2018).

17 Krug, K. et al. A Curated Resource for Phosphosite-specific Signature Analysis. Molecular \& cellular proteomics : MCP 18, 576-593, doi:10.1074/mcp.TIR118.000943 (2019).

18 Acosta-Jaquez, H. A. et al. Site-specific mTOR phosphorylation promotes mTORC1-mediated signaling and cell growth. Molecular and cellular biology 29, 4308-4324, doi:10.1128/mcb.01665-08 (2009).

19 Zhang, F. et al. O-GlcNAc modification is an endogenous inhibitor of the proteasome. Cell 115, 715725, doi:10.1016/s0092-8674(03)00974-7 (2003). 
20 Burt, R. A. et al. Novel antibodies for the simple and efficient enrichment of native O-GlcNAc modified peptides. bioRxiv, 2021.2005.2028.446228, doi:10.1101/2021.05.28.446228 (2021).

21 Wulff-Fuentes, E. et al. The human O-GlcNAcome database and meta-analysis. Scientific data 8, 25, doi:10.1038/s41597-021-00810-4 (2021).

22 Renaud, L., Picher-Martel, V., Codron, P. \& Julien, J. P. Key role of UBQLN2 in pathogenesis of amyotrophic lateral sclerosis and frontotemporal dementia. Acta neuropathologica communications 7, 103, doi:10.1186/s40478-019-0758-7 (2019).

23 Hofmann, K. \& Bucher, P. The UBA domain: a sequence motif present in multiple enzyme classes of the ubiquitination pathway. Trends in biochemical sciences 21, 172-173 (1996).

24 Wilde, I. B., Brack, M., Winget, J. M. \& Mayor, T. Proteomic characterization of aggregating proteins after the inhibition of the ubiquitin proteasome system. Journal of proteome research 10, 1062-1072, doi:10.1021/pr1008543 (2011).

25 Hart, G. W. Nutrient regulation of signaling and transcription. The Journal of biological chemistry 294, 2211-2231, doi:10.1074/jbc.AW119.003226 (2019).

26 Swamy, M. et al. Glucose and glutamine fuel protein O-GlcNAcylation to control T cell self-renewal and malignancy. Nature immunology 17, 712-720, doi:10.1038/ni.3439 (2016).

27 Saxton, R. A. \& Sabatini, D. M. mTOR Signaling in Growth, Metabolism, and Disease. Cell 169, 361371, doi:10.1016/j.cell.2017.03.035 (2017).

28 Sodi, V. L. et al. mTOR/MYC Axis Regulates O-GlcNAc Transferase Expression and O-GlcNAcylation in Breast Cancer. Molecular cancer research : MCR 13, 923-933, doi:10.1158/1541-7786.mcr-14-0536 (2015).

29 Park, S., Pak, J., Jang, I. \& Cho, J. W. Inhibition of mTOR affects protein stability of OGT. Biochemical and biophysical research communications 453, 208-212, doi:10.1016/j.bbrc.2014.05.047 (2014).

30 Wu, D., Jin, J., Qiu, Z., Liu, D. \& Luo, H. Functional Analysis of O-GlcNAcylation in Cancer Metastasis. Frontiers in oncology 10, 585288, doi:10.3389/fonc.2020.585288 (2020).

$31 \mathrm{Mi}, \mathrm{W}$. et al. O-GlcNAcylation is a novel regulator of lung and colon cancer malignancy. Biochimica et biophysica acta 1812, 514-519, doi:10.1016/j.bbadis.2011.01.009 (2011).

32 Wang, L. et al. Suppressed OGT expression inhibits cell proliferation while inducing cell apoptosis in bladder cancer. BMC cancer 18, 1141, doi:10.1186/s12885-018-5033-y (2018).

33 Tian, T., Li, X. \& Zhang, J. mTOR Signaling in Cancer and mTOR Inhibitors in Solid Tumor Targeting Therapy. International journal of molecular sciences 20, doi:10.3390/ijms20030755 (2019).

34 de la Cruz López, K. G., Toledo Guzmán, M. E., Sánchez, E. O. \& García Carrancá, A. mTORC1 as a Regulator of Mitochondrial Functions and a Therapeutic Target in Cancer. Frontiers in oncology $\mathbf{9}$, 1373, doi:10.3389/fonc.2019.01373 (2019).

35 Kumatori, A. et al. Abnormally high expression of proteasomes in human leukemic cells. Proceedings of the National Academy of Sciences of the United States of America 87, 7071-7075, doi:10.1073/pnas.87.18.7071 (1990).

36 Arlt, A. et al. Increased proteasome subunit protein expression and proteasome activity in colon cancer relate to an enhanced activation of nuclear factor E2-related factor 2 (Nrf2). Oncogene 28, 3983-3996, doi:10.1038/onc.2009.264 (2009).

37 Chen, L. \& Madura, K. Increased proteasome activity, ubiquitin-conjugating enzymes, and eEF1A translation factor detected in breast cancer tissue. Cancer research 65, 5599-5606, doi:10.1158/00085472.can-05-0201 (2005). 
38 Manasanch, E. E. \& Orlowski, R. Z. Proteasome inhibitors in cancer therapy. Nature reviews. Clinical oncology 14, 417-433, doi:10.1038/nrclinonc.2016.206 (2017).

39 Dahlman, K. B. et al. Modulators of prostate cancer cell proliferation and viability identified by shorthairpin RNA library screening. PloS one 7, e34414, doi:10.1371/journal.pone.0034414 (2012).

40 Jantrapirom, S., Piccolo, L. L., Pruksakorn, D., Potikanond, S. \& Nimlamool, W. Ubiquilin Networking in Cancers. Cancers 12, doi:10.3390/cancers12061586 (2020).

41 Yoshida, K. et al. Clinical Significance of Ubiquitin-associated Protein 2-like in Patients With Uterine Cervical Cancer. In vivo (Athens, Greece) 34, 109-116, doi:10.21873/invivo.11751 (2020).

42 Latonen, L. et al. Amplification of the 9p13.3 chromosomal region in prostate cancer. Genes, chromosomes \& cancer 55, 617-625, doi:10.1002/gcc.22364 (2016).

43 He, J., Chen, Y., Cai, L., Li, Z. \& Guo, X. UBAP2L silencing inhibits cell proliferation and G2/M phase transition in breast cancer. Breast cancer (Tokyo, Japan) 25, 224-232, doi:10.1007/s12282-017-0820-x (2018).

$44 \mathrm{Li}, \mathrm{D}$. \& Huang, Y. Knockdown of ubiquitin associated protein 2-like inhibits the growth and migration of prostate cancer cells. Oncology reports 32, 1578-1584, doi:10.3892/or.2014.3360 (2014).

45 Scott, D. A. Analysis of Melanoma Cell Glutamine Metabolism by Stable Isotope Tracing and Gas Chromatography-Mass Spectrometry. Methods in molecular biology (Clifton, N.J.) 2265, 91-110, doi:10.1007/978-1-0716-1205-7_7 (2021).

$46 \mathrm{Li}, \mathrm{X}$. et al. Tet proteins influence the balance between neuroectodermal and mesodermal fate choice by inhibiting Wnt signaling. Proceedings of the National Academy of Sciences of the United States of America 113, E8267-E8276, doi:10.1073/pnas.1617802113 (2016).

47 Katrancha, S. M. et al. Trio Haploinsufficiency Causes Neurodevelopmental Disease-Associated Deficits. Cell reports 26, 2805-2817 e2809, doi:10.1016/j.celrep.2019.02.022 (2019).

48 Mertins, P. et al. Ischemia in tumors induces early and sustained phosphorylation changes in stress kinase pathways but does not affect global protein levels. Molecular \& cellular proteomics : MCP 13, 1690-1704, doi:10.1074/mcp.M113.036392 (2014).

49 Subramanian, A. et al. Gene set enrichment analysis: a knowledge-based approach for interpreting genome-wide expression profiles. Proceedings of the National Academy of Sciences of the United States of America 102, 15545-15550, doi:10.1073/pnas.0506580102 (2005). 
a
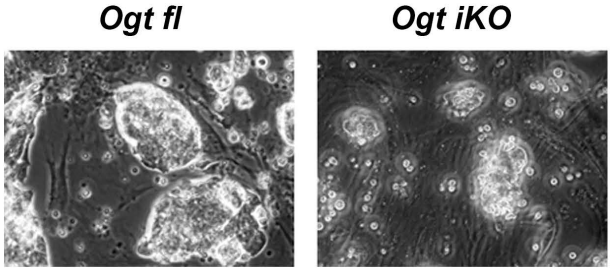

b

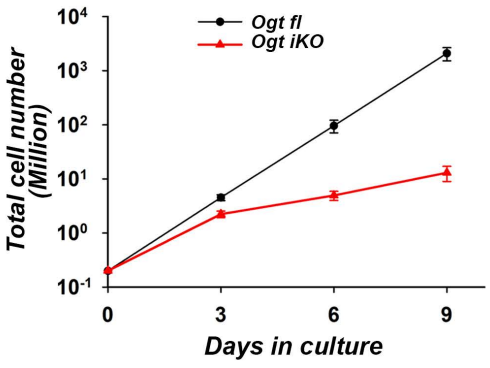

C

Day0

Day7

Day13

Genome-wide

CRISPR Library

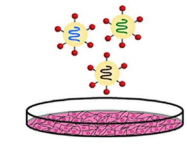

Ogt floxed mESCs

(expressing Cas9)

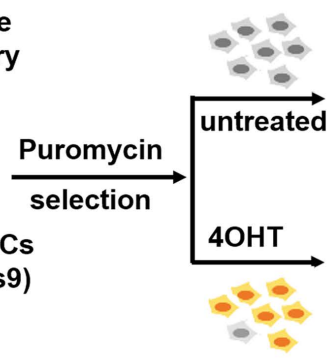

d $\circ$ Rep1 only 215 hits

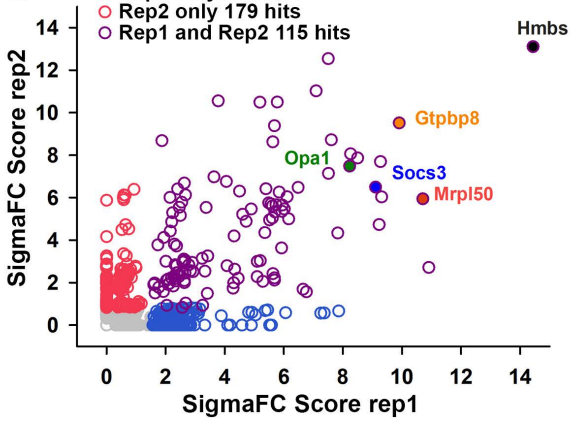

e

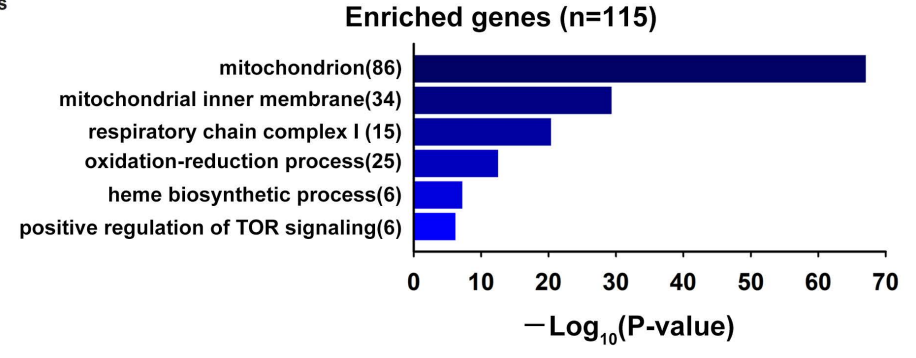

f

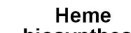

biosynthesis

PRC2
OEzh2 Ocnot9 Ouros OUrod OCpox $_{\text {OPtpap }}^{\text {OHmbs }}$

OFbxw7

OPde12 mTOR OMios

OCoa7
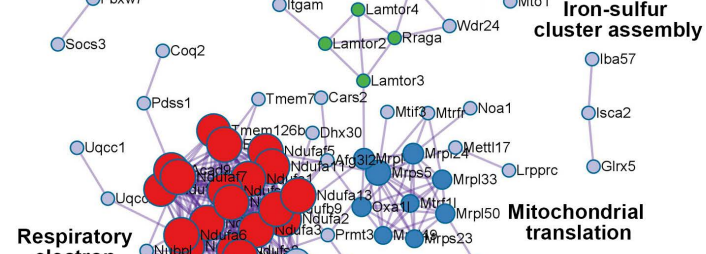

Resp

spiratory

electron

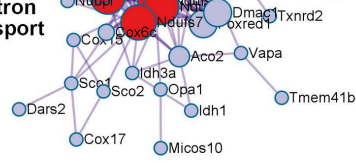

Ndufa13
Nffug
Nduag translation

OMrm3

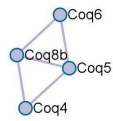

Quinone biosynthesis
Sequencing and sgRNA distribution analysis
Ogt floxed

(YFP-)

Ogt iKO

(YFP+)

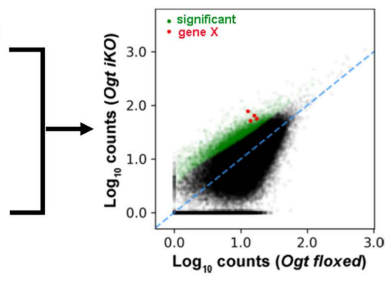

espiratory chain complex I(15)

(6)

heme biosynthetic process(6)

g

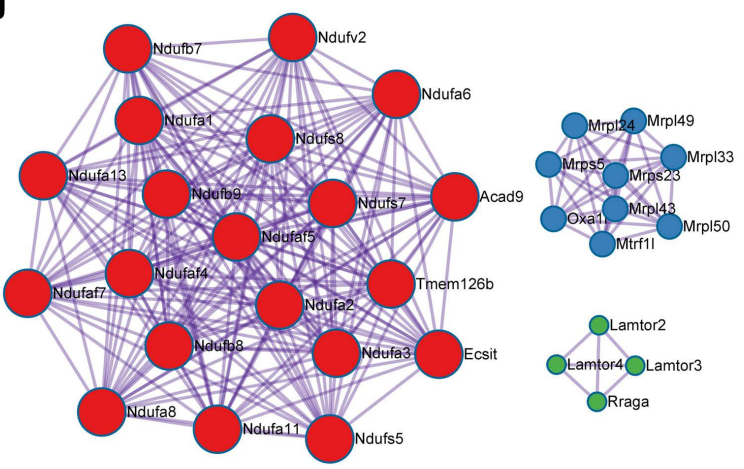

MCODE_1 Respiratory electron transport

MCODE_2 Mitochondrial translation

MCODE_3 mTOR signalling 
a

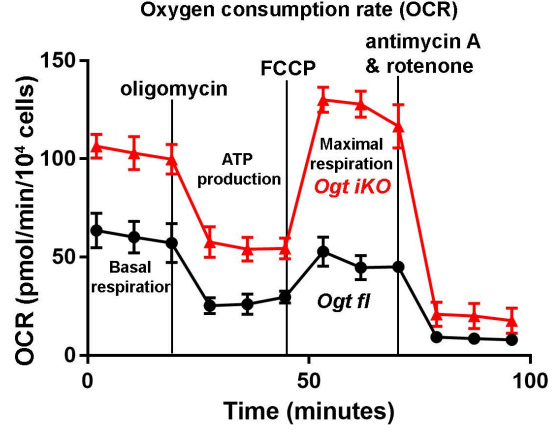

C
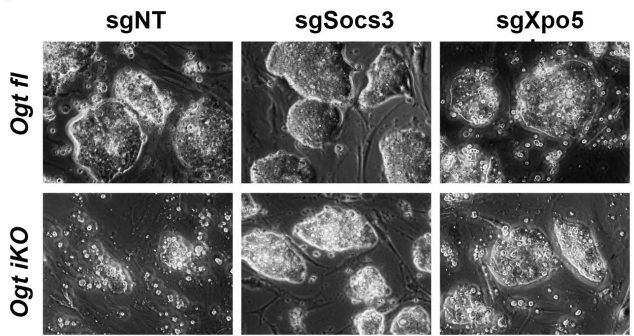

b

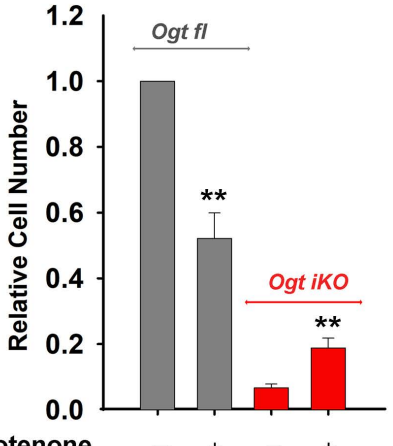

Rotenone d

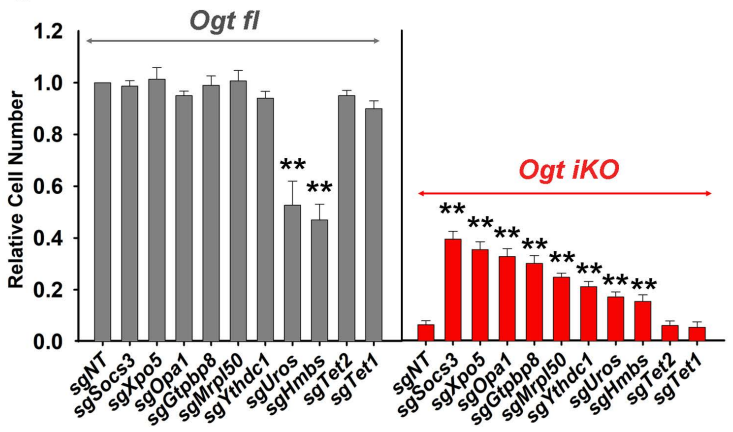

e

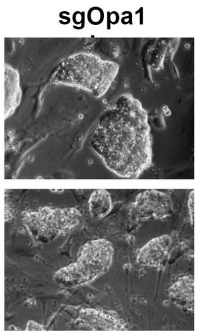

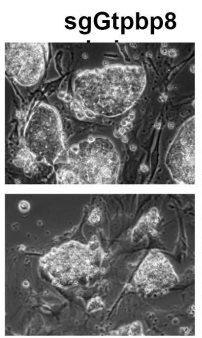

sgMrpI50
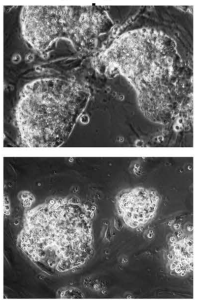

f

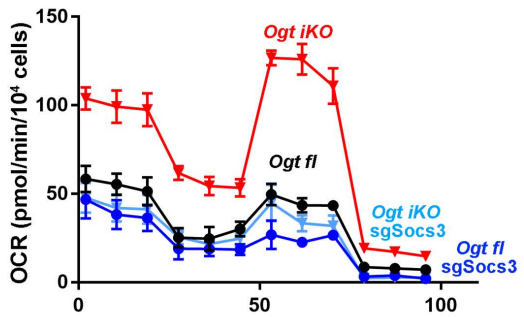

Time (minutes)

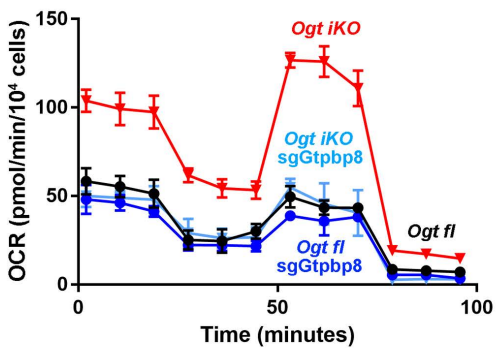

g

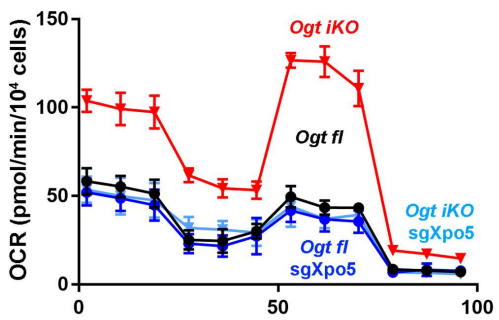

Time (minutes) 
a

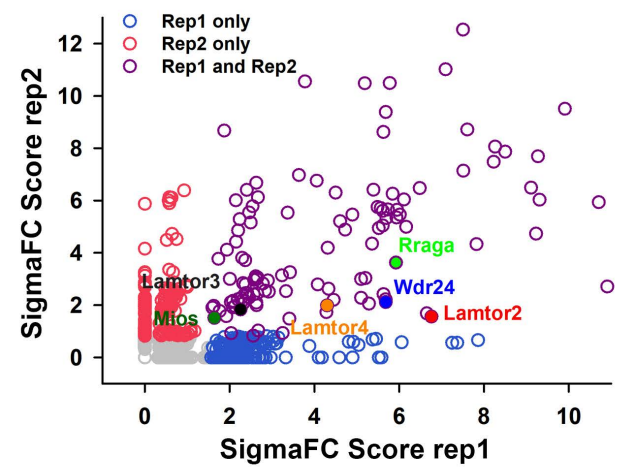

d

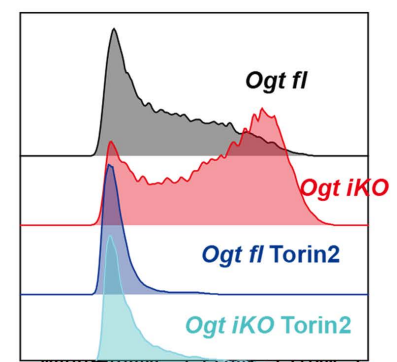

$\begin{array}{lllll}-10^{3} & 0 & 10^{3} & 10^{4} & 10^{5}\end{array}$

phospho-S6-AF555 $\longrightarrow$

f
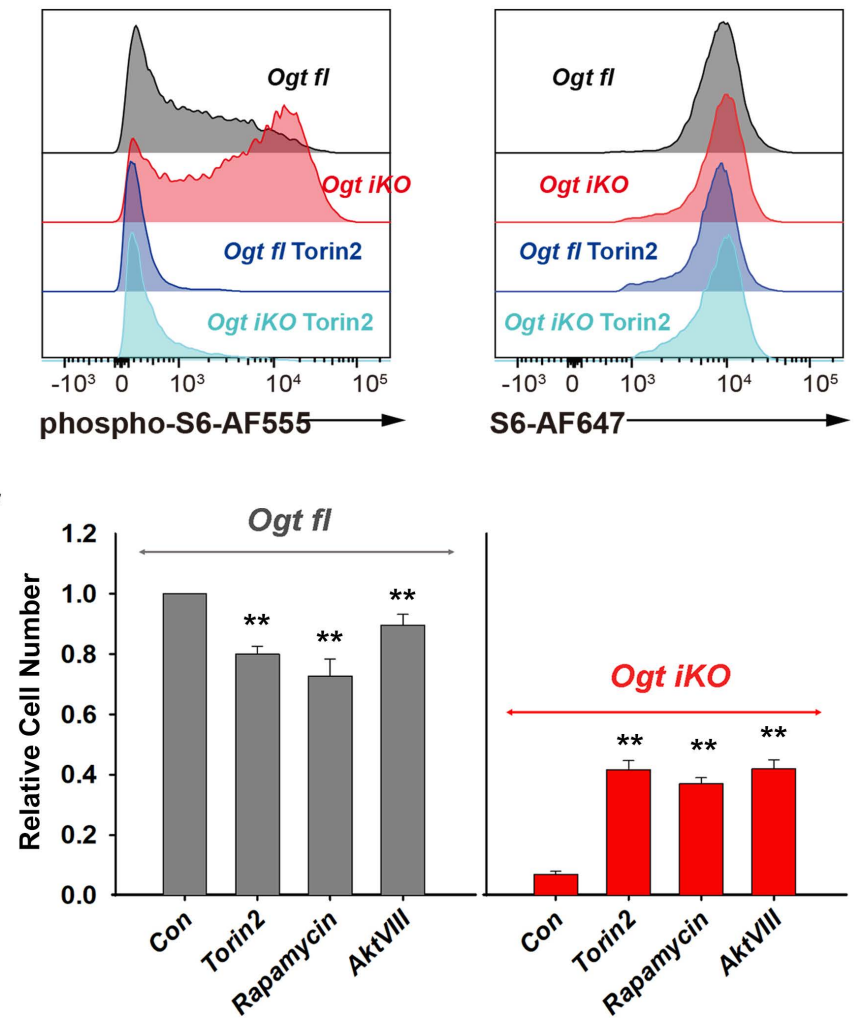

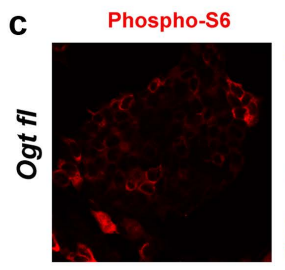

Phospho-S6/DAPI
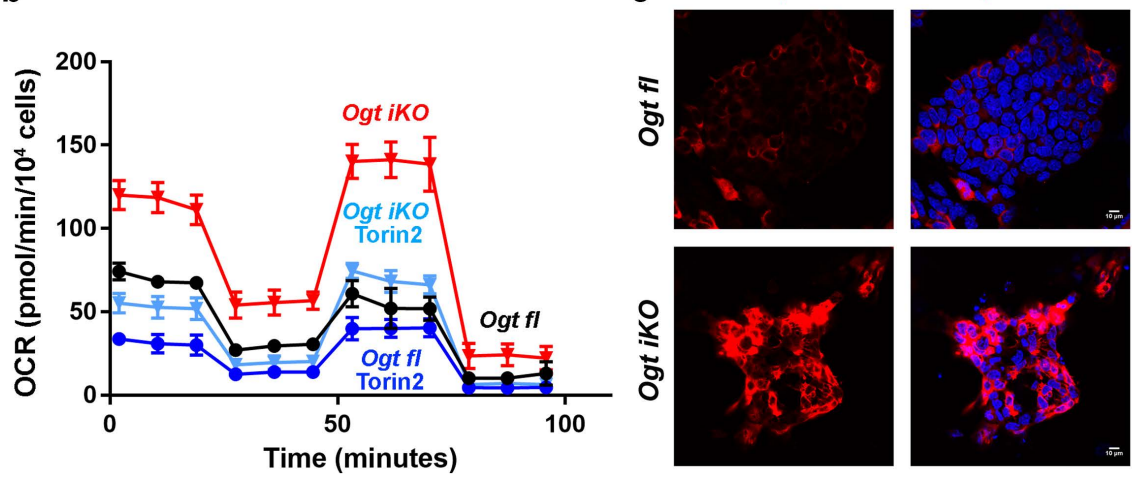

e

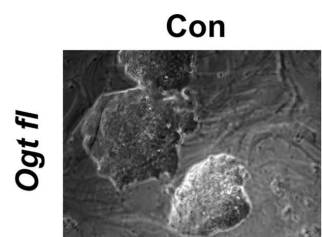

Torin2
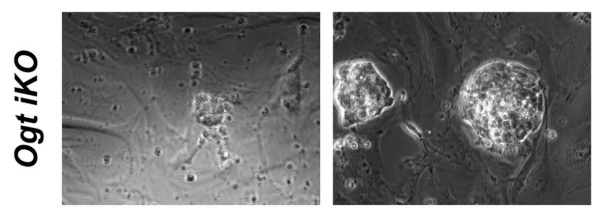

g

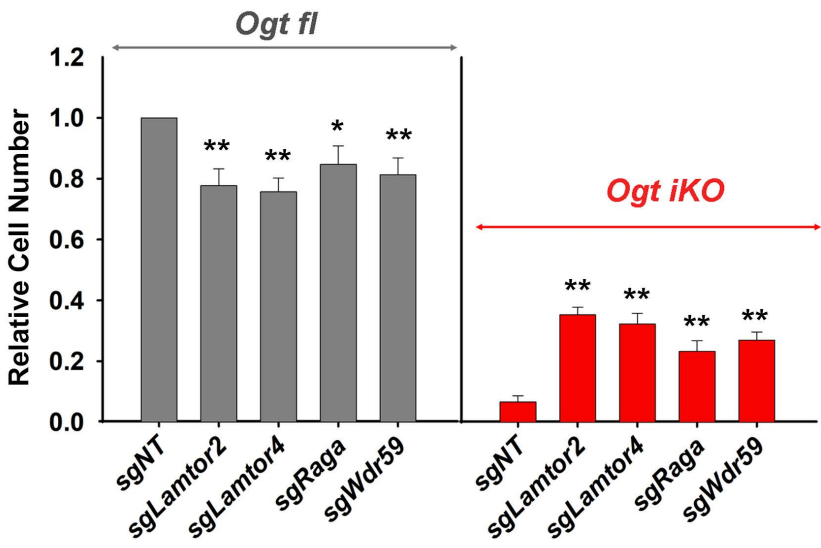




\section{fig 4}

a
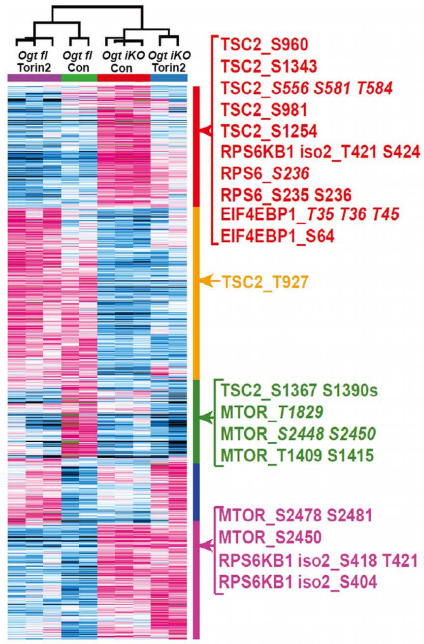

b

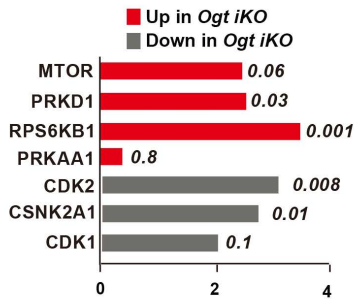

C

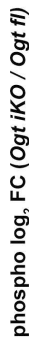

$-5$

phosphopeptide log2 FC $\mathrm{N}=16,457$

Normalized enrichment score Kinase Activity (mouse)

MTOR S2478 S2481

MTOR_S2450

RPS6KB1 iso2_S418 T421 S424 RPS6KB1 iso2_S404

RPS6_S235_S236

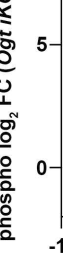

protein $\log _{2}$ FC (Ogt iKO / Ogt fl) d

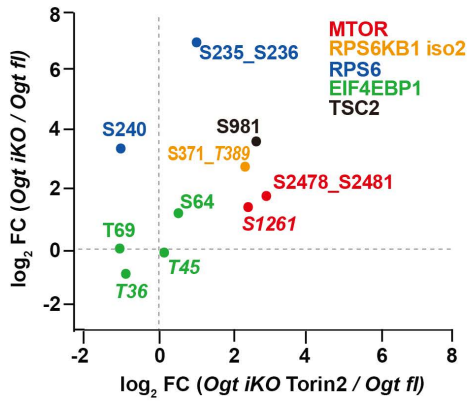

e

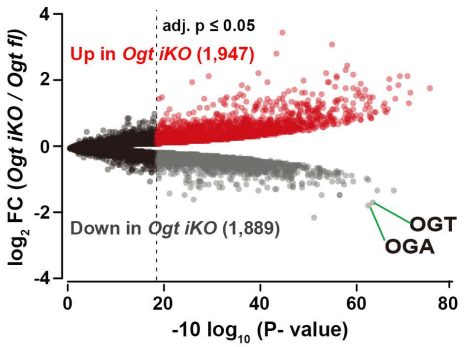

Hallmark Gene Set: PI3K-AKT-MTOR (NES=1.4; FDR=0.04)

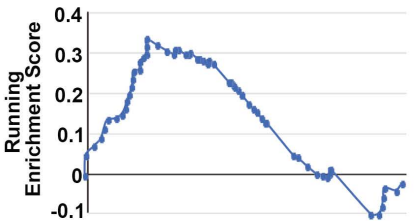

Rank in Gene List 
